\title{
Prenatal diagnosis of a particular limb body wall complex ${ }^{*}$
}

\author{
B. Fakhir, I. Jadid, A. Aboulfalah, H. Asmouki, A. Soummani \\ Department of Obstetrics and Gynecology, Mohamed VI University Hospital, Cadi Ayyad University, Marakesh, Morocco \\ Email: fakhirb@gmail.com
}

Received 25 December 2013; revised 22 January 2014; accepted 30 January 2014

Copyright (C) 2014 B. Fakhir et al. This is an open access article distributed under the Creative Commons Attribution License, which permits unrestricted use, distribution, and reproduction in any medium, provided the original work is properly cited. In accordance of the Creative Commons Attribution License all Copyrights (C) 2014 are reserved for SCIRP and the owner of the intellectual property B. Fakhir et al. All Copyright (C) 2014 are guarded by law and by SCIRP as a guardian.

\section{ABSTRACT}

Limb Body Wall Complex (LBWC) is a rare heterogenic group of fetal malformations including major defects of the ventral body (thoracoabdominal) wall associated with other anomalies including those of the limbs that may range from amelia to mild positional deformations, unusual craniofacial malformations, and a variety of visceral abnormalities that include the heart, lungs, genitourinary system. Tow phenotypes are described according to the feto-placental attachment. We report a particular case and iconography of LBWC associated with a giant sacral meningocele never described before. The patient was 26 years old, primigravida, with no illnesses or recent infections. She had no history of prenatal exposure to teratogenic agents, nor any family history of congenital malformations. She was referred for polymalformatif syndrome at $\mathbf{3 0}$ gestation weeks. Advanced sonography showed giant omphalocel with eviscerated liver, stomac and kidney, club feet, kyphoscoliosis and giant sacral fluid cyst. A 2100 g fetus was delivered, post natal examination confirmed LBWC with abdominoplacental attachment and the particularity of the presence of giant sacral meningocele, two bilateral hemi-genital tubercles and anal atresia.

\section{KEYWORDS}

Fetal Malformation; Laparoscisis; Feto-Placental Attachment; Kyphoscolisis

\section{INTRODUCTION}

Limb-body wall complex (LBWC) is a rare, complicated, polymalformative fetal malformation syndrome including neural tube defects, body wall disruption and limb ab-

\footnotetext{
*The case report was approved by gynecology obstetrics department ethics committee.
}

normalities [1]. The incidence at birth is about 0.32 per 100,000 births because the majority of affected fetuses undergo intrauterine deaths [2,3]. Pathogenesis is complicated and the possible pathogenetic mechanisms for LBWC include early amnion rupture, vascular disruption, and early embryonic maldevelopment. Generally the diagnosis is based on two of three of the following anomalies: Exencephaly or encephalocele with facial clefts, major defect of the ventral body wall (Thoraco and/or abdominoschisis) and Limbs anomalies that may range from amelia to mild positional deformations. Two phenotypes have been described, according to "placentocranial" or "placento-abdominal" adhesions [1,2]. Early ultrasonographic examination (12 weeks of amenorrhea) can make antenatal diagnosis [4]. Few cases are described, we present with iconography a case of LBWC with an interesting feature noted, consisting on the presence of a huge sacred meningocele never described before in the literature.

\section{CASE REPORT}

26 years old, primigravida, with no illnesses or recent infections. She had no history of prenatal exposure to teratogenic agents, nor any family history of congenital malformations. She was referred for polymalformatif syndrome at 30 gestation weeks. Advanced sonography showed giant omphalocel with eviscerated liver (Figure 1), stomach and kidney (Figure 2), club feet, kyphoscoliosis (Figure 3) and giant sacral fluid cyst. LBWC was suspected but the sacral cyst was an atypical association. A 2100 g still birth fetus was delivered prematurely and spontaneously, will decision was not yet taken. Post natal examination confirmed LBWC with abdomino-placental and amniotic attachment and the particularity was the presence of giant sacral meningocele, two bilateral hemi-genital tubercles and anal artesia. Unfortunately placenta was arrached during delivery but amniotic membranes was still attached to abdomen (Figures 4 (a)-(c)). 


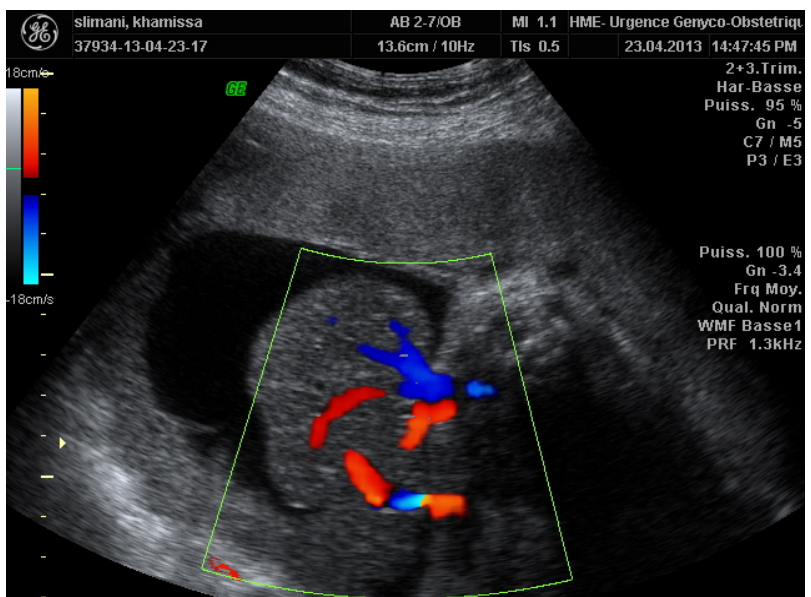

Figure 1. Colour flow imaging scans of the fetus showing a large ill defined abdominal wall defect through which the liver herniated into the extra embryonic coelom.

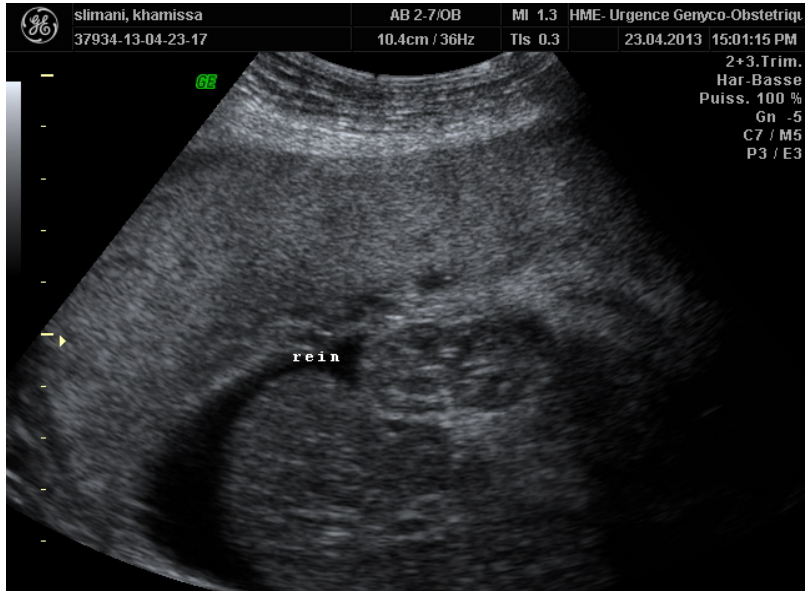

Figure 2. Ultrasound aspect with kidney protrusion through anterior abdominal defect.

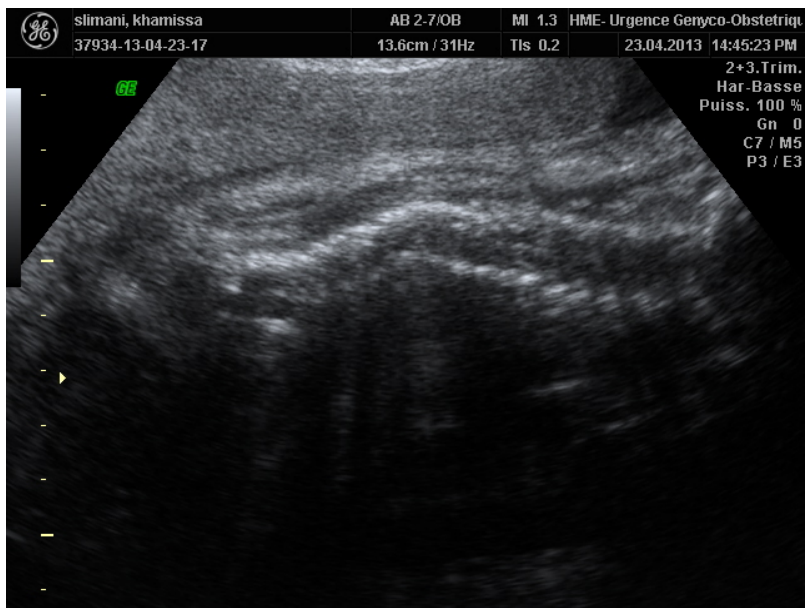

Figure 3. Ultrasound aspect of the fetal kyphoscoliosis.

Feotopathology and caryotype was not done because inability in our centre.

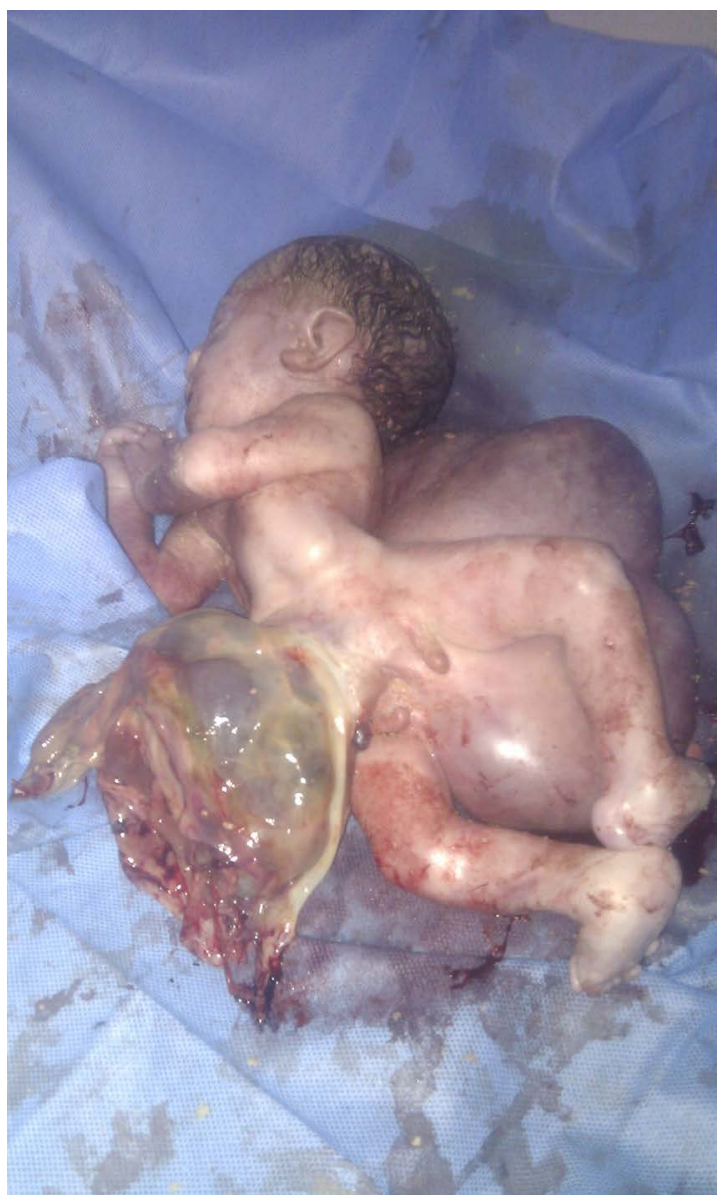

(a)

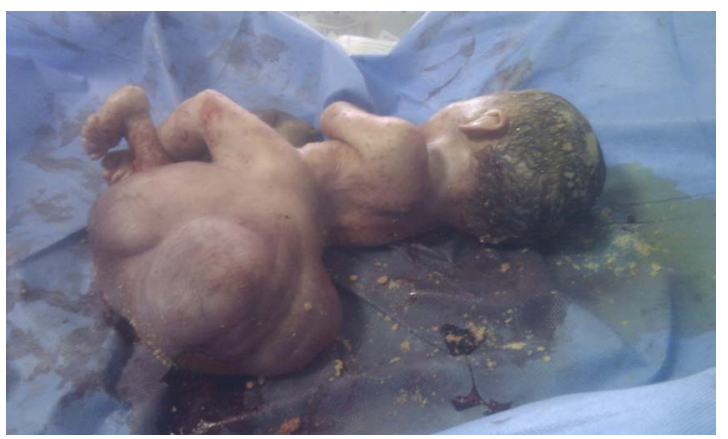

(b)

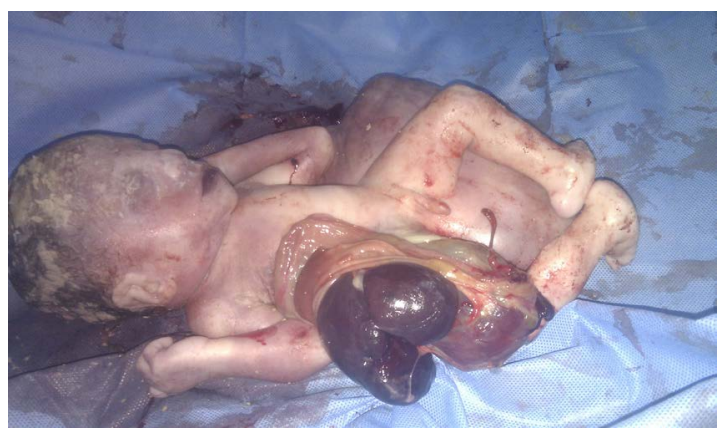

(c)

Figure 4. General appearance after delivery. 


\section{DISCUSSION}

The limb body wall complex is also known as the bodystalk syndrome [1]. It is a rare entity characterized by severe malformations. Most fetuses are aborted, either spontaneously or by medical means [5]. Most of them are stillborn. Postnatal survival for a significant duration is extremely rare, but at least one such case is reported; the child has severe physical handicaps [5]. This congenital anomaly has no sex or familial predilection or known recurrence risk. Caryotype study has been reported to be normal in all cases of LBWC. Van Allen [6,7], who described the syndrome for the first time in 1987 mentioned 3 essential features: 1) Exencephaly/encephalocele with facial clefts, 2) thoraco andabdominoschisis, 3) limb defect. In general, the diagnosis is based on tow of the above features. Different authors described the two different phenotypes, according to the placental attachment: placento-cranial and placento-caudal types.

1) The placento-cranial attachment, showing craniofacial defects and amniotic bands and/or adhesion; it seems to be related to early vascular disruption.

2) The placento-abdominal attachment that presents urogenital anomalies, anal atresia, short umbilical cord, abdominal placental attachment and persistence of anextraembrioniccelom. It seems to be due to an intrinsic embryonic maldevelopment.

Also, torpinet and all have suggested that LBWC results from amnion rupture caused by vascular or mechanical compression occurring between third and fifth weeks $[7,8]$. In our case report, the pattern of presentation resembled the "placento-abdominal" adhesion phenotype. Serum alpha-fetoprotein measurement and ultrasonographic examination are the key of prenatal diagnosis [9]. The ultrasound milestone of LBWC consists of thoraco-abdominal defect, spinal and cord abnormalities, positional limb deformities and abnormalities of umbilical cord and membranes. All these groups of defects were present in our case. The severity of the defects and a distorted appearance of the fetus make recognition of the fetal parts difficult in almost all cases of LBWC.

LBWC should be differentiated from common abdominal wall defects such as gastroschisis, omphalocele and uncommon entities like ectopiacordis, amniotic band syndrome, cloacal dystrophy and urachalcyst. There are also some specific signs for LBWC: the presence of membranes covering the contents of the herniated sac, any associated bowel abnormalities, the presence or absence of urinary bladder, scoliosis and limb defects [6, 9].
In our case, we report a huge sacred meningocele undescribed before in the literature.

\section{CONCLUSION}

LBWC is a rare polymalformative syndrome possible to detection with antenatal ultrasound scan. The syndrome is invariably fatal, but the couple must be reassured that there is no risk for recurrence. The diagnosis can be established early, thanks to the fascinating progress of sonographic technology and followed by medical termination of the pregnancy.

\section{CONFLICT OF INTEREST}

There is no conflict of interest.

\section{REFERENCES}

[1] Rachad, M., Chaara, H., Bouguern, H. and Melhouf, A. (2012) Le "limb body wall complex": A propos d'une observation assez particulière. The Pan African Medical Journal, 11, 20.

[2] Panduranga, C., et al. (2013) Limb body wall complex: A rare anomaly. Lab Physicians, 5, 65-67.

[3] Das, S.K., et al. (2013) The limb-abdominal wall complex defects, a form of amniotic band syndrome: A rare case report. Journal of Clinical and Diagnostic Research, 3, 543-544.

[4] Deruelle, P., Hay, R., Subtil, D., Chauvet, M.P., Duroy, A., Decocq, J. and Puech, F. (2000) Antenatal diagnosis of limb body wall complex. Journal de Gynecologie Obstetrique et Biologie de la Reproduction, 29, 385-391.

[5] Plakkal, N., Jacob, S.E., et al. (2008) Limb body wall complex in a still born fetus: A case report. Cases Journal, 1, 86. http://dx.doi.org/10.1186/1757-1626-1-86

[6] Chen, C.P., et al. (2009) Limb-body wall complex in one fetus of a dizygotic twin pregnancy conceived by egg donation, in vitro fertilization and embryo transfer: Prenatal diagnosis and literature review. Taiwan Journal of Obstetrics \& Gynecology, 48, 446-450.

[7] Torpin, R. (1965) Amniochorionic mesobleast fibrous strings and amniotic bands. American Journal of Obstetrics and Gynecology, 91, 65-75.

[8] Miller, M.E., Graham, J.M., Higginbottom, M.C. and Smith D.W. (1981) Comparaison related defects from early amnios rupture: Evidence mechanical teratogenesis. Journal of Pediatrics, 98, 292-297. http://dx.doi.org/10.1016/S0022-3476(81)80664-6

[9] Socolov, D., et al. (2009) Limb body wall complex-Case presentation and literature review. Romanian Journal of Legal Medicine, 17, 133-138. 